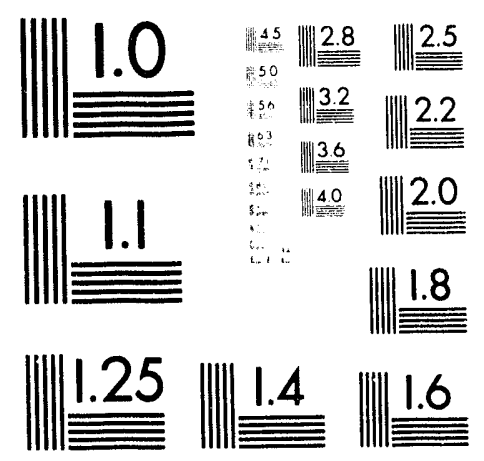



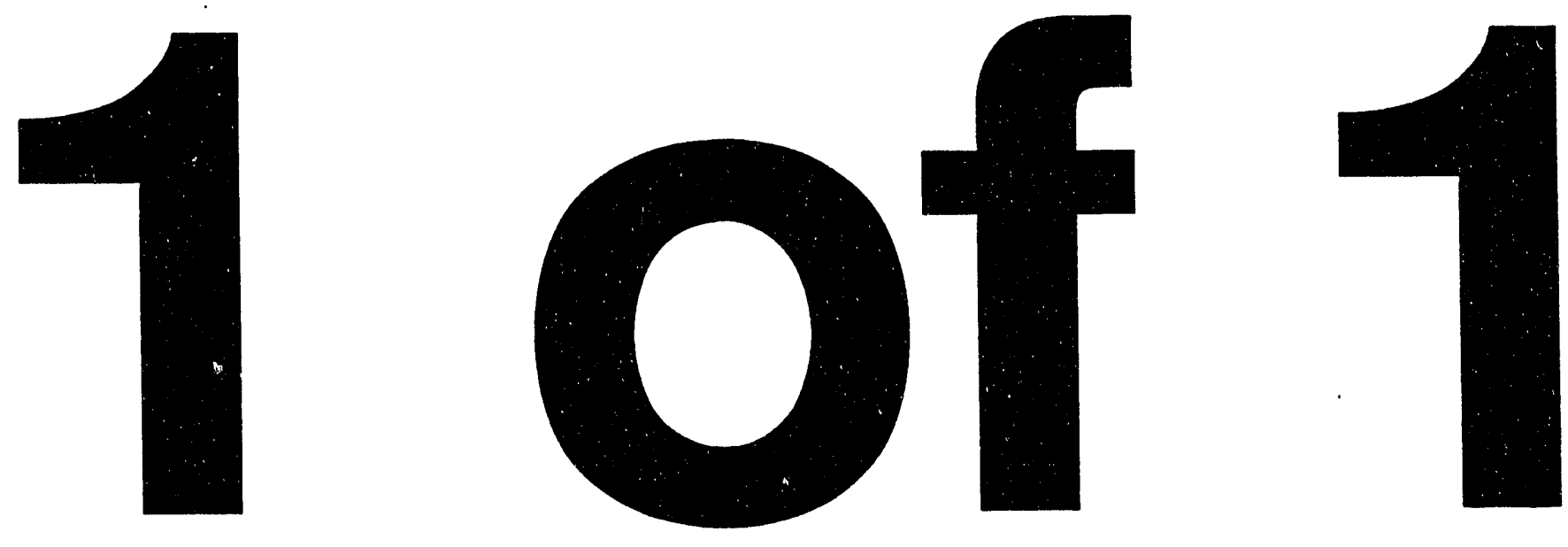


\title{
A Possible Redesign of the SLAC SLC Damping Rings*
}

\author{
T. O. RAUBENHEIMER, R. EARLY, 'T. LIMBERG, H. MOSIIAMMER, J. SPENCER \\ Stanford Linear Accelerator Center, Stanford University, Stanford, CA 94309
}

\begin{abstract}
We describe a possible replacement for the SLC damping rings that would generate bearns with normalized horizontal emittances of $\gamma \epsilon_{x}=9 \times 10^{-6} \mathrm{~m}-\mathrm{rad}$ when uncoupled; this is more than three times smaller than that generated by the current rings. The primary difference between the new design and the current ring is the arc cell structure; the insertion regions, the kickers, and the RF are essentially unchanged. The new cell uses a single combined function bending magnet, roughly $70 \mathrm{~cm}$ in length, to replace the two bends, defocusing quadrupole, and defocusing sextupoles in the current FODO cell; the focusing quadrupole and sextupoles, used in the current cell, are also used in the new structure. The length of the new cell is identical to that of the current cell and thus nine of these new cells would simply replace the nine FODO cells in each arc of the rings.
\end{abstract}

\section{Introduction}

In this paper, we will describo a possible replacement for the Stanford Linear Accelerator (SLC) damping rings. The SLC damping rings [1] are very compact storage rings, operating at $1.19 \mathrm{GeV}$ with a circumference of 35.28 meters. They have transverse damping times of $3.5 \mathrm{~ms}$ and equilibrium rms emittances of $\gamma c_{x}=3 \times 10^{-5} \mathrm{~m}$-rad. The rings are composed of 18 FODO bending cells plus two insertion regions for injection/extraction, kickers, and $R F$.

Although the equilibrium emittance of the SLC damping rings is very small, given the recent performance of the SLC, we can consider the possibility of transporting even smaller emittances to the IP [2]. The ninimum emittance at the IP is set by the synchrotron radiation emittance growth through the SLC arcs. In normalized units, this is roughly $\gamma \epsilon_{x} \approx 1.3 \times 10^{-5}$ and $\gamma \epsilon_{y} \approx 0.1 \times 10^{-5} \mathrm{~m}$-rad. These emittances will he added to the emittances at the end of the SLC linac and thus we used these values as a goal for the emittances of a future damping ring; one has diminishing returns when achieving values significantly smalier.

We will first discuss the constraints on the replacement ring and then we will describe the lattice and the bending magnets. Next, we describe the dynamic aperture limitations and a possible correction scheme, and finally, we discuss other possible upgrades to the SLC damping rings.

\section{Emittance Reduction and Lattice Choice}

When considering a replacernent, one is constrained to the same energy, the same, or faster, damping times, and roughly the same circumference as the SLC damping

* Work supported by Department of Energy, contract DEAC03-76SF00515.

Presented at the Particle Accelerator Conference (PAC 93), Washington rings. The encrgy is constrained by the required rotation of the electron polarization before injection into the ring. the damping times are needed to damp the injected emittances while operating at $120 \mathrm{~Hz}$, and the circumference is determined by the size of the damping ring vaults. Thus, there are only three possible ways of reducing the emittances: (1) use damping wigglers to decrease both the damping times and the emittances, (2) use combined function bending magnets to change the horizontal damping partition, also decreasing the damping times and the emittances, and (3) use a more efficient cell structure with smaller dispersion to reduce the quantum excitation and thus the emittance.

Unfortunately, wigglers are not very effective at reducing the emittance. Roughly four meters of $5 \mathrm{~T}$ superconducting wiggler with a $15 \mathrm{~cm}$ period would be needed to reduce the damping time and emittance by a factor of two. In addition to the severe operational difficulties with such a device, the wigglers would incre sse the longitudinal emittance an unacceptable amount.

This leaves the latter two choices, changing the damping partitions and/or using a more emittance efficient lattice. We chose a combination: increasing the horizontal damping partition to roughly 1.7 while decreasing the quantum excitation in the cells. Many emittance efficient cell structures have been developed such as the (hasmanGreen, Triple Bend Achromat (TBA), and Theoretical Minimum Emittance ('TME) structure. Unfortunately, all of these structures require more physical space than is available; they are not very compact. For example, 12 Chasman-Green cells, using 24 bending magnets, could be used to generate the desired emittance, but to keep the quadrupole strengths reasonable, we found that the ring circumference would have to double. Alternately, 16 TME. cells, using 16 bending magnets, could also generate the desired emittance, but again the ring circumference nearly doubled.

Thus, we chose to use a FODO type structure where all of the vertical focusing is performed in a combined function bending magnet located between the horizontally focusing quadrupoles; we refer to this as a FOOF structure. $[3,4]$ The cell structure is similar to the TME structurc in that the horizontal dispersion and horizontal beta function are minimum at the center of the bending magnet and, witl the parameters we have chosen, the equilibrium emittances are comparable; 20 FOOF cells are needed to achieve the same emittance as 16 TME cells. This structure was actually suggested as a replacement for the SLC damring rings in 1989, but this option was not studied seriously until recently.

\section{Lattice}

The new ring is illustrated schematically in the bottom half of Fig. 1 while the current SLC rings are shown in the top half. As described, the FOOF lattice structure 


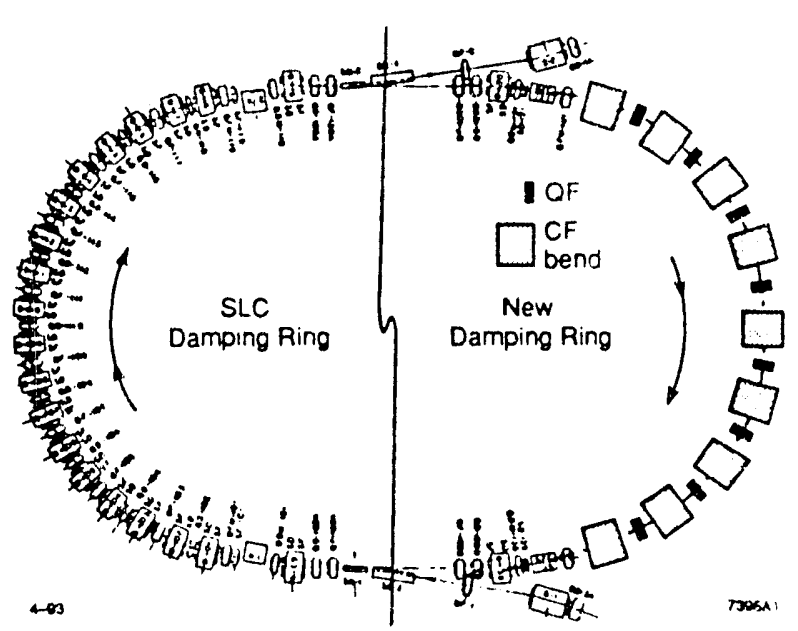

Fig. 1 Schematic of the current SLC damping ring (upper half) and a new damping ring design (lower half). The arc cells of the SLC rings are replaced in the new design while the injection/extraction, $R F$, and kicker regions remain the same.

Table 1. Damping ring parameters.

\begin{tabular}{|c|c|c|c|c|c|}
\hline & $\widehat{B_{0}}[\mathrm{kG}]$ & $\tau_{x}[\mathrm{~ms}]$ & $J_{x}$ & $\tau_{y}[\mathrm{~ms}]$ & $\gamma \epsilon_{x}[\mathrm{~mm}-\mathrm{mrad}]$ \\
\hline SLC & 20.24 & 3.5 & 1.0 & 3.5 & 30 \\
\hline new & 18.34 & 2.0 & 1.7 & 3.5 & 9 \\
\hline
\end{tabular}

consists of a focusing quadrupole and a defocusing combined function bending magnet. The cell length chosen to be equal to that of the SLC damping ring FODO cells, 1.29 meters, and the cell tunes were chosen close to those of the current rings: $\nu_{x} \approx 0.37$ and $\nu_{y} \approx 0.13$; this is close to the optimal focusing to minimize the emittance.

Thus, nine of the new arc cells simply replace nine of the original FODO arc cells. The original focusing quadrupoles are reused and the injection/extraction insertion regions are not changed. The chromatic correction is performed using the focusing permanent magnet sextupoles in the present rings and a defocusing sextupole gradient in the bending magnets. Thus, a single combined function bending magnet, roughly twice the length of the present bending magnets, replaces two bends, a defocusing quadrupole, and the defocusing sextupoles. Additional, dipole correctors and trim quadrupoles are needed for orbit correction and control of the tunes and a new vacuum chamber is required.

The lattice functions of the new cells and the original FODO cells are compared in Figs. 2 and 3. The beta functions are similar in the two cells, but the dispersion, and thus the quantum excitation, is significantly smaller in the new cell. Parameters of the present SLC damping rings and the new damping ring are listed in Table 1 . The equilibrium emittance in the new ring is over a factor of three smaller than that in the present rings. This is due
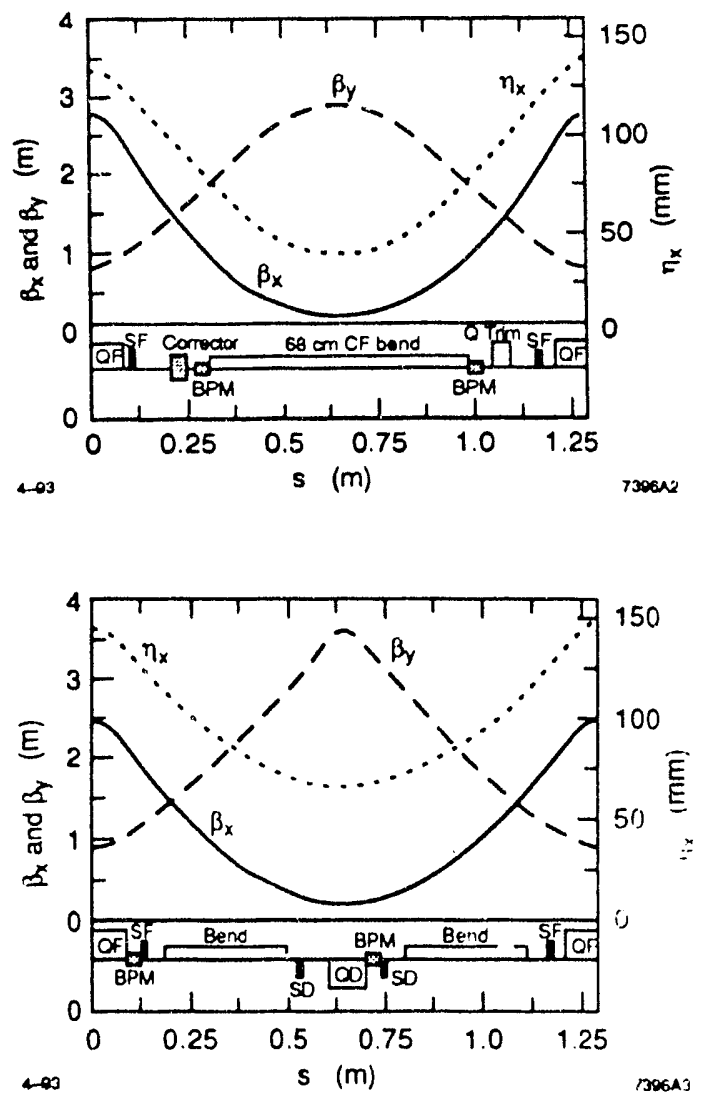

Figs. 2 and 3 Lattice functions in an arc cell of the new damping ring (top) and the SLC rings (bottom); the cell length is the same in both.

to both the change in the damping partition from the defocusing gradient in the bending magnets and the smaller dispersion in the bending magnets.

\section{Combined Function Bending Magnet}

The combined function bending magnet is described in detail in Ref. 5 . It is $68 \mathrm{~cm}$ long with fields of $B_{0}=18.33$ $\mathrm{kG}, d B_{y} / d x=-140 \mathrm{kG} / \mathrm{m}$, and $d^{2} B_{y} / d x^{2}=-4770$ $\mathrm{kG} / \mathrm{m}^{2}$. The gap was chosen to be equal to the aperture in the focusing quadrupoles, $1.3 \mathrm{~cm}$ radius, which is larger than the current bending magnets; this would allow a constant radius vacuum chamber without the aperture transitions that can increase the ring impedance.

To achieve good fields without differential saturation of the magnet pole, we chose to design the bending magnet with Vanadium Permendur poles. This allows one to change the ring energy without changing the normalized quadrupole and sextupole components of the bend. Another advantage of the long bends with permendur poles is that the fringe fields are small. The SLC bending magnets are constructed from saturated iron and are relatively short and, although the peak field is roughly $20.2 \mathrm{kG}$, the fringe fields reduce the rms bending field to less than $18 \mathrm{kG}$ 


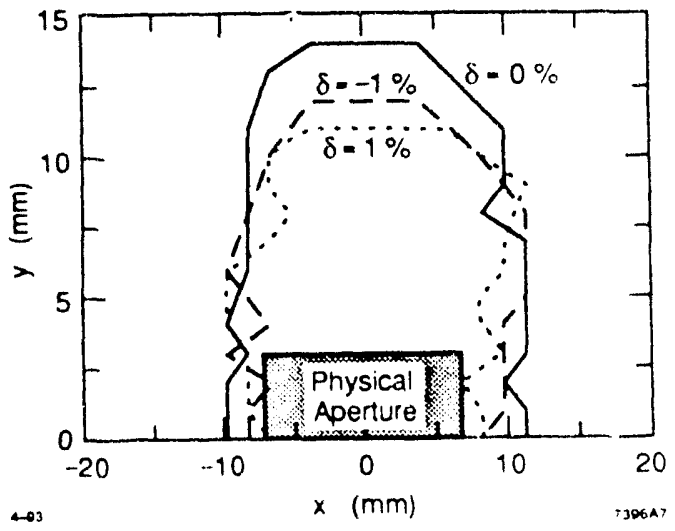

Fig. 4 Dynamic aperture in the new damping ring design at the injection point for initial energy deviations of $0 \%$ and $\pm 1 \%$. Particles were tracked for 1000 turns with synchrotron oscillations and alignment errors.

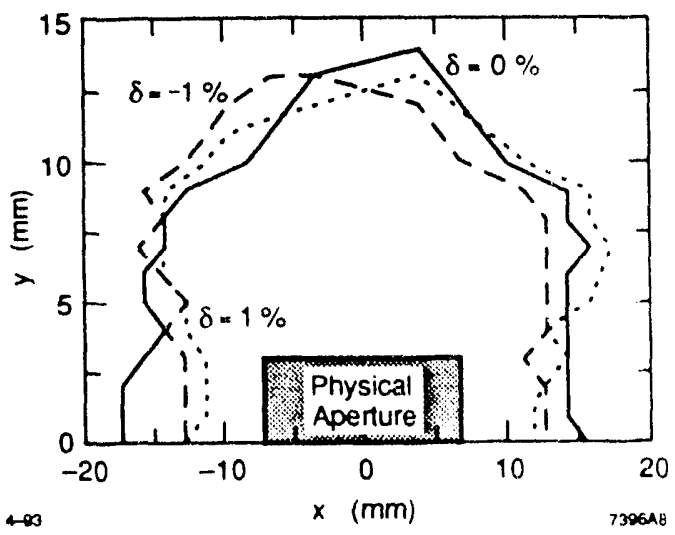

Fig. 5 Dynamic aperture in the new damping ring with an octupole field of $K_{3} L=1000 \mathrm{~m}^{-3}$ added to each focusing quadrupole; synchrotron oscillations and alignment errors were included.

Thus, the synchrotron radiation power and therefore the vertical damping times from the SLC and the new bending magnets are roughly equal, even though the SLC magnets have peaks fields that are $10 \%$ higher.

\section{Dynamic Aperture}

The dynamic aperture in the new ring is slightly poorer than in the current SLC rings. This is arises because of the additional sextupole field that is needed to correct the chromaticity since the dispersion is smaller and because of the octupole-like terms that arise in the combined function bending magnet. Despite the reduction, the dynamic aperture is still larger than the physical aperfure. This is illustrated in Fig. 4 where the aperture was calculated with the alignment and field errors and synchrotron oscillations.
Although this aperture should be sufficient, it is possible to increase it by adding octupoles; this is true for both the SLC and the new damping ring. The prime limitation on the aperture is the horizontal tune shift with amplitude. This can be corrected with octupoles fields in the focusing quadrupoles. The dynamic aperture is plotted in Fig. 5 where an integrated octupole field of $1000 \mathrm{~m}^{-3}$ was added to the QF magnets; alignment and field errors and synchrotron oscillations were included. This octupole field could be generated with either permanent magnets or by shimming the quadrupole poles 10 mils.

\section{Alternate Upgrades}

There are two primary problems with the design we have described: time and money. There are two upgrades that may be more attractive since they are much faster, cheaper and less disruptive to implement even though they only offer half the emittance gain. These require modifying the present SLC bending magnets to change the horizontal damping partition to roughly 1.7. The dispersion is not changed, but, with the additional damping, the horizontal emittance is reduced by a factor of 1.7 . The damping partitions could be changed by adding a gradient to the bends or by changing the edge focusing of the bending magnets.

\section{Conclusions}

We have described a possible upgrade for the SLC damping rings which entails replacing the present bending magnets with longer combined function magnets. The new rings have the same circumference and generate the same arnount of synchrotron radiation as the present rings, but have an equilibrium horizontal emittance that is a factor of three smaller. The emittance is reduced by increasing the horizontal damping partition and by reducing the quantum excitation in the bending magnets. Finally, we are pursuing two other possible upgrades. Although they only achieve half the emittance reduction, they are cheaper and easier to implement. These will be described in subsequent reports.

\section{References}

[1] G. Fischer, W. Davies-White, 'T. Fieguth, H. Wiedemann, "A 1.2 GeV Damping Ring Complex for the" Stanford Linear Collider," Proc. of the XIIth Int. Conf. on High Energy Acc., Fermilab, IL (1983).

[2] C. Adolphsen, et. al., "Flat Beams in the SLC," contributed to this conference.

[3] This lattice had been independently studied by J. P. Delahaye of CER N and T. Raubenheimer of SLAC in 1988 and was discussed at the "Workshop on Future Linear Colliders" at SLAC in December 1988; it is being used in the KEK ATF damping ring project [4].

[4] J. Urakawa, et. al., "The Damping Ring of Accelerator Test Facility for Linear Collider," Proc. of the XVth Int. Conf. on High Energy Acc., Hamburg, Germany (1992).

[5] R. Early and T. Raubenheimer, "Computer Studies of a Combined Function Bend Magnet," contributed to this conference. 

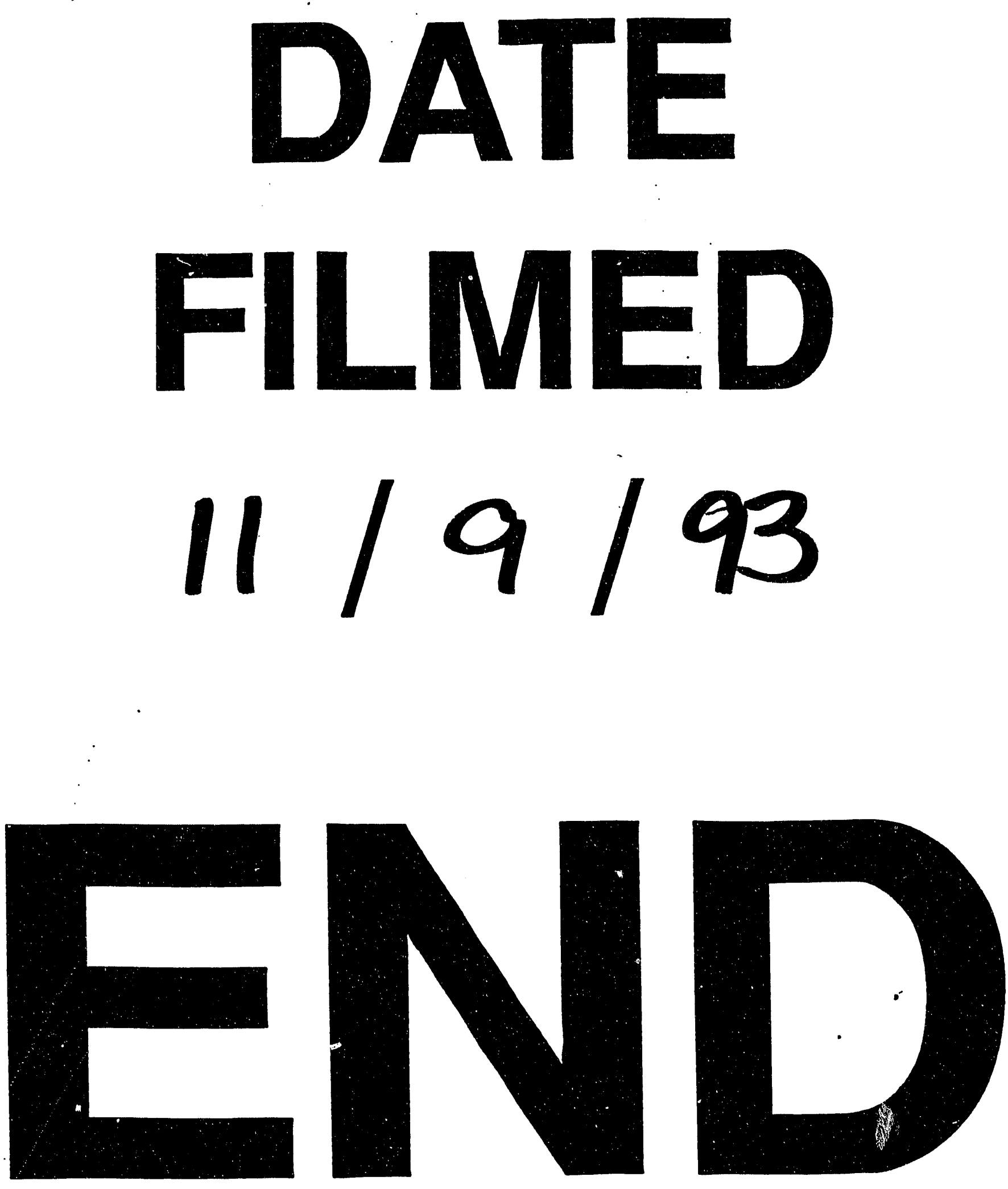
